\title{
Observability-singularity manifolds in the context of chaos based cryptography
}

\author{
Octaviana Datcu $^{1}$, Roger Tauleigne ${ }^{2}$, Adriana Vlad ${ }^{3}$ and Jean-Pierre Barbot ${ }^{4}$
}

\begin{abstract}
In the '80s Takens formulated the conditions that ensure the capability to reconstruct the dynamics of a transmitter when an observer receives one scalar output from the transmitter. In practical situations, the reconstruction of the original system is strongly influenced by the choice of the variable transmitted over the communication channel. This paper aims to analyze this influence in the context of mathematical singularities occurring in the evolution of the chaotic manifolds used in encryption. We analyze two systems having a chaotic behavior, a discrete recurrence, the HitzlZele map, and a continuous system, the Colpitts oscillator. We show the existence of observability singularities in both cases. The numerical experiments show that the dynamics of the discrete system falls in these singularities sets, but very infrequently. More surprisingly, the dynamics of the continuous system can not pass through the singularity, which is situated at infinity. But an exponential factor allows the chaotic dynamics to approach the vicinity of the singularity better than $10^{-7}$ and that, for about $30 \%$ of its duration. The noise inherent in analog signals are much higher than this value, the observation of the system is impossible in practice. For an effective application to data encryption, it will be helpful to increase the duration during which the dynamics remains in the vicinity of the singularity.
\end{abstract}

\section{PROBLEM STATEMENT}

The context of the present work is the chaos-based enciphering. Let us choose a didactical example, the generalized Hénon map, [7], which has the analytical expression given by equations (1), as underlying system of a chaos-based cipher.

The used enciphering method is the inclusion; see, for example, [9] for a practical realization of the inclusion method. Therefore, the message $m$ is included in the first equation of system (1) and evolves simultaneously with the dynamics of the generalized Hénon chaotic system.

$$
\begin{aligned}
& x_{1}^{+}=a-x_{2}^{2}-b \cdot x_{3} \\
& x_{2}^{+}=x_{1} \\
& x_{3}^{+}=x_{2}
\end{aligned}
$$

where $a$ and $b$ are parameters chosen so that chaotic behavior is engendered. The notation $x_{i}^{+}$is chosen to express the future state in the evolution of the dynamical system, $x_{i}^{+}=$ $f_{i}\left(x_{i}\right), x_{i}^{j+}=f^{(j)}\left(x_{i}\right) ; i=\{1,2,3\}$.

\footnotetext{
${ }^{1}$ O. Datcu is with ETTI, Politehnica University of Bucharest, 313 Splaiul Independentei, Romania octavianadatcu at yahoo.com

${ }^{2} \mathrm{R}$. Tauleigne is with ENSEA, 6 Avenue du Ponceau, 95014, CergyPontoise, France tauleigne at ensea.fr

${ }^{3}$ A. Vlad is with Research Institute for Artificial Intelligence, Romanian Academy, Calea 13 Septembrie, Bucharest, Romania and ETTI, Politehnica University of Bucharest, 313 Splaiul Independentei, Romania avlad at racai.ro

${ }^{4}$ J.-P. Barbot is with ENSEA, 6 Avenue du Ponceau, 95014, CergyPontoise, France barbot at ensea.fr
}

The chaotic dynamics of the generalized Hénon map is not the goal of our work. Nevertheless, the reader may refer to [7], in order to investigate more on this issue. Here, our main purpose is to deal with observability-singularity [2].

At the end level of the transmission, to recover the dynamics of the transmitter, an observer is used. The observer must dispose of sufficient measurements of the output $y$ of the transmitter (2). As the message is added to the first state $x_{1}$ of the system (1), the third state $x_{3}$ is chosen to be the output of the transmitter, as it has the greatest relative degree to the secret input, i.e. $x_{3}$ is the furthest from $x_{1}$ in the phase space. Refer to [10] and [18] for a more complex discussion about relative degrees in a chaotic context.

$$
\begin{aligned}
x_{1}^{+} & =a-x_{2}^{2}-b \cdot x_{3}+m \\
x_{2}^{+} & =x_{1} \\
x_{3}^{+} & =x_{2} \\
y & =x_{3}
\end{aligned}
$$

Each series of three values of the output of system (2), $Y=\left(x_{3}, x_{3}^{+}, x_{3}^{2+}\right)^{T}$, the output and its upcoming iterations, ensures, at the reception, the reconstruction of all the states of the transmitter, $x_{2}=x_{3}^{+}, x_{1}=x_{3}^{2+}, x_{3}=x_{3}$. In order to recover also the message, from the series of values of the output, the knowledge of a fourth value, $x_{3}^{3+}=$ $x_{2}^{2+}=x_{1}^{+}=a-x_{2}^{2}-b \cdot x_{3}+m$, is necessary. Roughly speaking, the left invertibility problem means to recover the input from the output knowledge; see [1] and [16] for more details. Let us focus to the reconstruction of the states of the transmitter. At the reception the series of values $\left\{x_{3}, x_{3}^{+}, x_{3}^{2+}, \ldots\right\}$ is received. The reconstruction of all the states of the transmitter lies on solving the system (3), where $X$ is the column vector of the state variables of the system (1).

$$
M \cdot X=Y \Leftrightarrow\left[\begin{array}{lll}
0 & 0 & 1 \\
0 & 1 & 0 \\
1 & 0 & 0
\end{array}\right]\left[\begin{array}{l}
x_{1} \\
x_{2} \\
x_{3}
\end{array}\right]=\left[\begin{array}{c}
x_{3} \\
x_{3}^{+} \\
x_{3}^{2+}
\end{array}\right]
$$

Cramer's rule, [11], although not necessary in this simple case, is used to solve the system, as in (4). 


$$
\begin{aligned}
& x_{1}= \frac{\left|\begin{array}{ccc}
x_{3} & 0 & 1 \\
x_{3}^{+} & 1 & 0 \\
x_{3}^{2+} & 0 & 0
\end{array}\right|}{|M|} ; x_{2}=\frac{\left|\begin{array}{ccc}
0 & x_{3} & 1 \\
0 & x_{3}^{+} & 0 \\
1 & x_{3}^{2+} & 0
\end{array}\right|}{|M|} ; \\
& x_{3}=\frac{\left|\begin{array}{ccc}
0 & 0 & x_{3} \\
0 & 1 & x_{3}^{+} \\
1 & 0 & x_{3}^{2+}
\end{array}\right|}{|M|}
\end{aligned}
$$

where $|M|=-1$ is the determinant of the matrix $M$.

It results from (4) that the dynamics at the reception is given by $x_{1}=x_{3}^{2+} ; x_{2}=x_{3}^{+} ; x_{3}=x_{3}$.

We focus on the nonlinear systems, more particularly on the case when the determinant of the matrix $M$ is null (but not always null), which is equivalent, in terms of cryptography, to the system being non-observable. The observability of nonlinear continuous time system (more precisely, locally weakly observability) is well known since the seminal work of Hermann and Krener [8]. For nonlinear systems three important facts are to be highlighted. First, contrarily to the linear case, there do not exist stopping criteria (the Cayley Hamilton theorem can not be invoked in nonlinear). So, the state information can be embedded in very far derivatives, for continuous time systems, and in very far upcoming outputs, for discrete time systems. Secondly, observability may depend of the input, [6] and, finally, the observability in nonlinear is only local. The first and last facts may be useful in order to improve the security of data transmission by inclusion method. Refer to [3] for the algebraic frame of these properties. As, in general, for the nonlinear case, the observability matrix $O$ has its coefficients depending on the states of the transmitter, the situation discussed here is when the system is observable, unless, in certain regions of the phase space, called singularityobservability manifolds, where the determinant of the matrix $O$ becomes zero. Investigations were done in [13] and [5] for the quality of the reconstruction of the state portrait of the transmitter, i.e., its states, depending on the chosen measured variable of the transmitter.

\section{OBSERVABILITY SINGULARITY MANIFOLDS}

A system is said to be observable when, by measuring the sequence of values of one of the states of the system, the entire phase space of the system can be reconstructed, under a suitable smooth transformation. The singularity observability manifold $S_{\bar{O}}$ of a chaotic system is the mathematical space in which, seen from the measured variable, the system losses its observability property. Some definitions are given, for the observability notions, in the case of the discrete-time hyperchaotic Rössler map, in [4].

Let us consider a nonlinear discrete system described in the three-dimensional space $\mathbb{R}^{3}$, with $x=\left(x_{1}, x_{2}, x_{3}\right)^{T} \in$ $\mathbb{R}^{3}$ the state vector evaluated at the $j$-th iteration. Let the evolution of the system at the next iteration be $x_{i}^{+}=$ $x_{i}(j+1) ; i=1,2,3$. Using the sequence of values of the observable $x_{i}$ and its subsequent iterations up to the $(n-1)-t h$ order, it is possible to reconstruct the entire phase space of the system that produced the measured state. In the considered case, $n=3$ denotes the dimension of the involved attractor. The coordinate change between the original threedimensional real phase space $\left(x_{1}, x_{2}, x_{3}\right)^{T}$ and the iterative embedding $(U, V, W)^{T} \in \mathbb{R}^{3}$ is defined by equations (5).

$$
\phi_{i}:\left\{\begin{array}{l}
U=x_{i} \\
V=x_{i}^{+} \\
W=x_{i}^{++}
\end{array}\right.
$$

The observability matrix $O_{i}$ of a nonlinear system observed from the perspective of the $i$-th state variable of the system is the Jacobian of the application $\phi_{i}$, as defined in [15] and in [12] for continuous systems. The observability matrix $O_{i}$ is then expressed by equations (6).

$$
O_{i}=\left[\begin{array}{lll}
\partial U / \partial x_{1} & \partial U / \partial x_{2} & \partial U / \partial x_{3} \\
\partial V / \partial x_{1} & \partial V / \partial x_{2} & \partial V / \partial x_{3} \\
\partial W / \partial x_{1} & \partial W / \partial x_{2} & \partial W / \partial x_{3}
\end{array}\right]
$$

The studied system is said to be non-fully observable when the determinant of the observability matrix is null over some subspace of the phase space. The observability singularity manifold $S_{\bar{O}}$ is the subspace where the map $\phi_{i}$ cannot be inverted. The mathematical expression of this hyper-surface is given in equation (7).

$$
S_{\bar{O}, i}=\left\{\left(x_{1}, x_{2}, x_{3}\right) \in \mathbb{R}^{3}\left|\operatorname{det}\left(O_{i}\right)=\right| \Delta x_{i} \mid=0\right\}
$$

where $i$ is the number of the state variable that is measured.

The quality of the chosen measured variable is considered, here, to be a function of the existence of the singularities and their intersection with the attractor of the considered transmitter. While the strange attractor which characterizes the transmitter does not intersect the observability singularity manifolds, the vector of its space states can be reconstructed at the receiver by measuring only one state, the output of the transmitter, the observable. When the observed variable leads to an embedding which has singularity areas, the transmitter is no more observable when its characteristic strange attractor intersects these regions- the singularity observability manifolds. In order to decipher a secret message included in the evolution of the strange attractor, the complete space state must be reconstructed at the reception in any moment of the transmission. On the singularity observability manifolds, one is not able to reconstruct the secret message without error. These manifolds are to be exploited in cryptography in order to confuse a potential enemy who intercepts the secret transmission. The parameters of the chaotic system are periodically switched and exchanged between the communication partners. Having the complete knowledge of the parameters and initial conditions of the chaotic transmitter, the receiver is able to know when an observability singularity manifold is intersected by the strange attractor. The enemy does not have this information- parameters and initial conditions- so, he will continue to decipher, getting an erroneous space state, and implicitly, a false message. 


\section{A. Case study. The Hitzl-Zele map.}

Let us consider the discrete-time three dimensional chaotic system described by equations (8), in [17]. The vector of the bifurcation parameters is given by known $(a, b)$ for the analysis of the observability singularity manifolds induced by each of the state variables of the system.

$$
\begin{aligned}
& x_{1}^{+}=1+x_{2}-x_{3} \cdot x_{1}^{2} \\
& x_{2}^{+}=a \cdot x_{1} \\
& x_{3}^{+}=b \cdot x_{1}^{2}+x_{3}-0.5
\end{aligned}
$$

We consider the measured variable to be $x_{1}$. The coordinate transformation between the original phase space and the iterative embedding, and its corresponding observability matrix $O_{1}$, are given by the expressions in (9), and (10), respectively.

$$
\begin{gathered}
\phi_{1}:\left\{\begin{array}{l}
U=x_{1} \\
V=x_{2}-x_{3} \cdot x_{1}^{2}+1 \\
W=x_{2}^{+}-x_{3}^{+} \cdot x_{1}^{+2}+1
\end{array}\right. \\
O_{1}=\left[\begin{array}{ccc}
1 & 0 & 0 \\
-2 x_{1} x_{3} & 1 & -x_{1}^{2} \\
E_{1} & E_{2} & E_{3}
\end{array}\right]
\end{gathered}
$$

Its determinant is $\left|\left(O_{1}\right)\right|=\Delta_{x_{1}}=x_{1}^{2} E_{2}+E_{3}$. Substituting $E_{2}=\frac{\partial W}{\partial x_{2}}$ and $E_{3}=\frac{\partial W}{\partial x_{3}}$ in the expression of the determinant of the matrix $O_{1}$, the result in equation (11) is obtained for the observability singularity manifold computed from the observable $x_{1}$.

$$
\begin{aligned}
S_{\bar{O}, 1} & =\left\{\left(x_{1}, x_{2}, x_{3}\right) \in \mathbb{R}^{3} \mid 2 x_{1}^{4}\left(x_{3}^{2}-b+1\right)-\right. \\
& \left.-2 x_{1}^{2} x_{3}\left(x_{2}+1\right)+x_{2}\left(x_{2}+2\right)-1=0\right\}
\end{aligned}
$$

When the measured state variable is $x_{2}$, the coordinate transformation between the original phase space and the iterative embedding is given by the expression in (12). The corresponding observability matrix is then $\mathrm{O}_{2}$ described by equations (13). The equation describing the observability singularity manifold is thus given by (14).

$$
\begin{gathered}
\phi_{2}:\left\{\begin{array}{l}
U=x_{2} \\
V=a \cdot x_{1} \\
W=a \cdot\left(1+x_{2}-x_{3} \cdot x_{1}^{2}\right)
\end{array}\right. \\
O_{2}=\left[\begin{array}{ccc}
0 & 1 & 0 \\
a & 0 & 0 \\
-2 a x_{1} x_{3} & a & -a x_{1}^{2}
\end{array}\right] \\
S_{\bar{O}, 2}=\left\{\left(x_{1}, x_{2}, x_{3}\right) \in \mathbb{R}^{3} \mid x_{1}=0\right\}
\end{gathered}
$$

When the measured state variable is $x_{3}$, the coordinate transformation between the original phase space and the iterative embedding is given by the expression in (15). The corresponding observability matrix is then $O_{3}$ described by equations (16).

$\phi_{3}:\left\{\begin{array}{l}U=x_{3} \\ V=b \cdot x_{1}^{2}+x_{3}-0.5 \\ W=b \cdot x_{1}^{2}+x_{3}+b \cdot\left(1+x_{2}-x_{3} \cdot x_{1}^{2}\right)^{2}-1\end{array}\right.$

$$
O_{3}=\left[\begin{array}{ccc}
0 & 0 & 1 \\
2 b x_{1} & 0 & 1 \\
E_{1} & E_{2} & E_{3}
\end{array}\right]
$$

The determinant of the observability matrix is $\Delta_{x_{3}}=2 \cdot b$. $x_{1} \cdot E_{2}$. The expression of the derivative $E_{2}=\partial W / \partial x_{2}=$ $2\left(1+x_{2}-x_{1}^{2} x_{3}\right)$. Consequently, the observability singularity manifold is given by (17).

$$
\begin{aligned}
S_{\bar{O}, 3} & =\left\{\left(x_{1}, x_{2}, x_{3}\right) \in \mathbb{R}^{3} \mid x_{1}\left(1+x_{2}-x_{1}^{2} x_{3}\right)=0\right\} \\
& =S_{\bar{O}, 2} \cup\left\{\left(x_{1}, x_{2}, x_{3}\right) \in \mathbb{R}^{3} \mid 1+x_{2}-\right. \\
& \left.-x_{1}^{2} x_{3}=0\right\}
\end{aligned}
$$

This last condition takes $S_{\bar{O}, 2}$ and adds an additional constraint. As we will experimentally show, this condition is reached in chaotic regime. This recursive system falls in the observability singularity surfaces sets.

\section{B. Case study. The Colpitts chaotic oscillator.}

For the chaotic system expressed by (18), minutely described in [14], when choosing the states $x_{1}$ or $x_{2}$ as the output of a transmitter, the singularity observability manifolds are given in (19), and (20), respectively. The parameter $k$ does not influence the chaotic behavior and is kept fixed at $k=0.5$.

$$
\begin{aligned}
& \dot{x}_{1}=A\left(e^{-x_{2}}+1+x_{3}\right) \\
& \dot{x}_{2}=A \cdot x_{3} \\
& \dot{x}_{3}=-(k / A)\left(x_{1}+x_{2}\right)+B x_{3}
\end{aligned}
$$

where $A=g /[Q(1-k)]=g / Q k$ and $B=-1 / Q$.

$$
\begin{array}{r}
S_{\bar{O}, 1}=\left\{\left(x_{1}, x_{2}, x_{3}\right) \in \mathbb{R}^{3} \mid\right. \\
\left.A^{3} x_{3} e^{-x_{2}}\left(e^{-x_{2}}+A x_{3}+B\right)+A k=0\right\} \\
S_{\bar{O}, 2}=\left\{\left(x_{1}, x_{2}, x_{3}\right) \in \mathbb{R}^{3} \mid-k \cdot A=0\right\}
\end{array}
$$

If the output is considered to be the third state of the system (18), then:

$$
\phi_{3}:\left\{\begin{array}{l}
U=x_{3} \\
V=\dot{x}_{3}=-\frac{k}{A}\left(x_{1}+x_{2}\right)+B x_{3} \\
W=\ddot{x}_{3}=-\frac{k}{A}\left(\dot{x}_{1}+\dot{x}_{2}\right)+B \dot{x_{3}}
\end{array}\right.
$$

which results in an observability matrix having the expression in (22). 


$$
O_{3}=\left[\begin{array}{ccc}
0 & 0 & 1 \\
-k / A & -k / A & B \\
E_{1} & E_{2} & E_{3}
\end{array}\right]
$$

The determinant $\Delta x_{3}$ is, thus, equal to $\frac{k}{A}\left(E_{1}-E_{2}\right)$. As, $E_{1}=\frac{\partial W}{\partial x_{1}}=-\frac{k B}{A}$ and $E_{2}=\frac{\partial Z}{\partial x_{2}}=k\left(e^{-x_{2}}-\frac{k B}{A}\right)$, the observability-singularity manifold is, thus, given by equation (23).

$$
S_{\bar{O}, 3}=\left\{\left(x_{1}, x_{2}, x_{3}\right) \in \mathbb{R}^{3} \mid-\frac{k^{2}}{A} e^{-x_{2}}=0\right\}
$$

Since $x_{2}<\infty$, the system can not reach the singularity observability. But, the xalues of $x_{2}$ explored by the chaotic behavior approach better than $10^{-7}$ the condition of observability loss. In the analog systems, such as the Colpitts oscillator, this difference is much smaller than the noise of the analog signals. The trajectory of the attractor is then in the shadow of the singularity, in the sense of non-standard analysis. As long as that situation lasts the system is not observable.

\section{EXPERIMENTAL RESULTS}

\section{A. The Hitzl-Zele map}

We choose two pairs of parameters $(a, b)((0.275,0.87)$ and $(-0.69,0.85))$ for the chaotic Hitzl-Zele map. We compute Lyapunov exponents in order to establish that the chosen pairs engender chaotic behavior. Lyapunov exponents are plotted in Fig.1 for fixed $b=0.87$. Phase portraits are depicted in Fig. 2 for the two pairs $(a, b)$; one can observe the two distinct strange attractors.

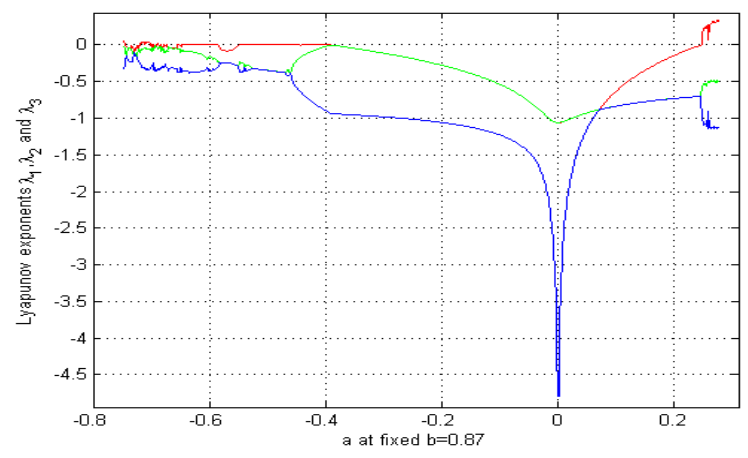

Fig. 1. Lyapunov exponents for the Hitzl-Zele map for fixed $b=0.87$.

We are interested if and how the singularity observability manifolds intersect the strange attractors for the chosen bifurcation parameters $a$ and $b$. To characterize on a single graph a large number of iterations, we have chosen to count the values of $\Delta x_{1}$ and $\Delta x_{3}$ in classes. All these values correspond to the chaotic trajectory. The number of occurrences for the values determinants $\Delta x_{1}$ and $\Delta x_{3}$, defined in equations (10) and (16), take on the strange attractors shown in Fig.2 are given in Fig.3. It can be observed that the singularity observability manifolds shift with the change of parameters $a$ and $b$. While for $(a, b)=(0.275,0.87)$,
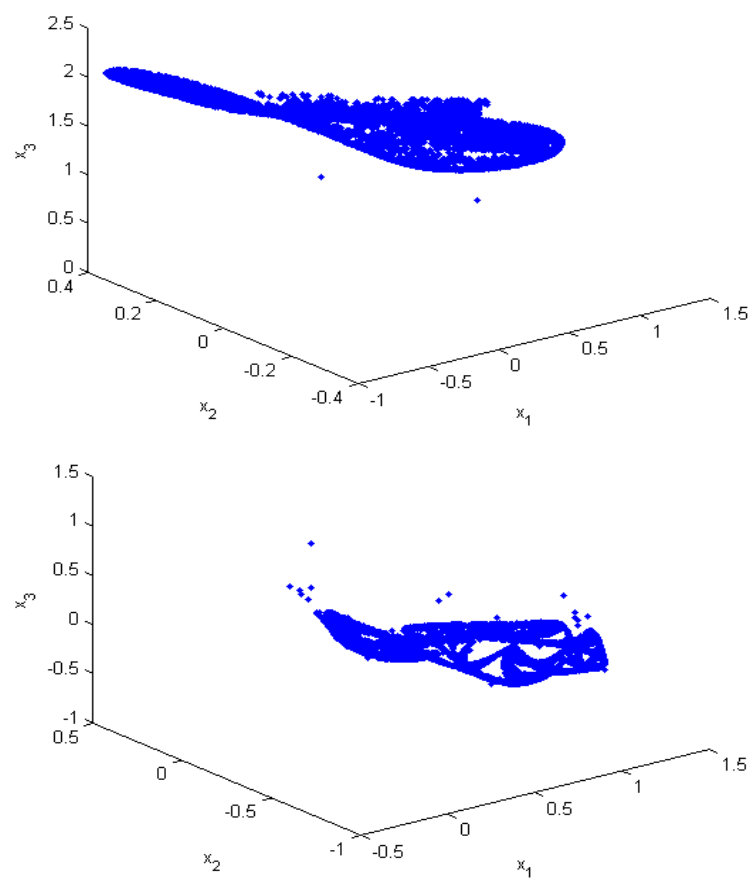

Fig. 2. Phase portrait for the Hitzl-Zele map for $\left(x_{0}(0), x_{2}(0), x_{3}(0)\right)=$ $(0.65,0.17,0.7)$. The parameters are $(a, b)=(0.275,0.87)$ (up) and $(a, b)=(-0.69,0.85)($ down $)$.

$\Delta x_{1}$ never becomes zero, for $(a, b)=(0.275,0.87)$ it passes through the observability singularity. For the second state variable chosen as observable, the two sets of parameters $(a, b)$ lead to similar distributions of the determinant of the observability matrix. For the output $y=x_{3},(a, b)=$ $(-0.69,0.85)$ characterizes a strange attractor which falls in the singularity manifold set described by $\Delta x_{3}=0$ apparently more times than for the other choice of $a$ and $b$.

The numerical exploration of this recurrence reveals that extremely few iterations approach the observability singularity surface. Observed following the state $x_{2}$, only 700 values of $\Delta x_{2}$ are smaller than $10^{-6}$, a ratio of $0.175 \%$. Observed following the state $x_{3}$, this ratio is even smaller, $0.0025 \%$. These results show that the existence of an observability singularity surface is not sufficient to guarantee the use in data encryption; it is still necessary that the dynamics of the system explores it long enough to actually interrupt the transmitter-receiver synchronization.

\section{B. The Colpitts oscillator.}

For the Colpitts oscillator we take the state $x_{3}$ as observable. The bifurcation parameters are kept at fixed values $g=4.46$ and $Q=1.38$. These values correspond to a chaotic behavior. In Fig. 6 we follow the evolution of the values the determinant $\Delta x_{3}$ corresponding to the choice of $y=x_{3}$, on the attractor generated by the given parameters and initial conditions. The sampling step is taken $T_{s}=10^{-3} \mathrm{~s}$.

From the theoretical point of view, the singularity is a plan in the phase space in $\mathbb{R}^{3}$. The trajectory of the strange attractor is a line in $\mathbb{R}^{3}$. The intersection between the singularity manifold and the strange attractor is, consequently, a dot. 

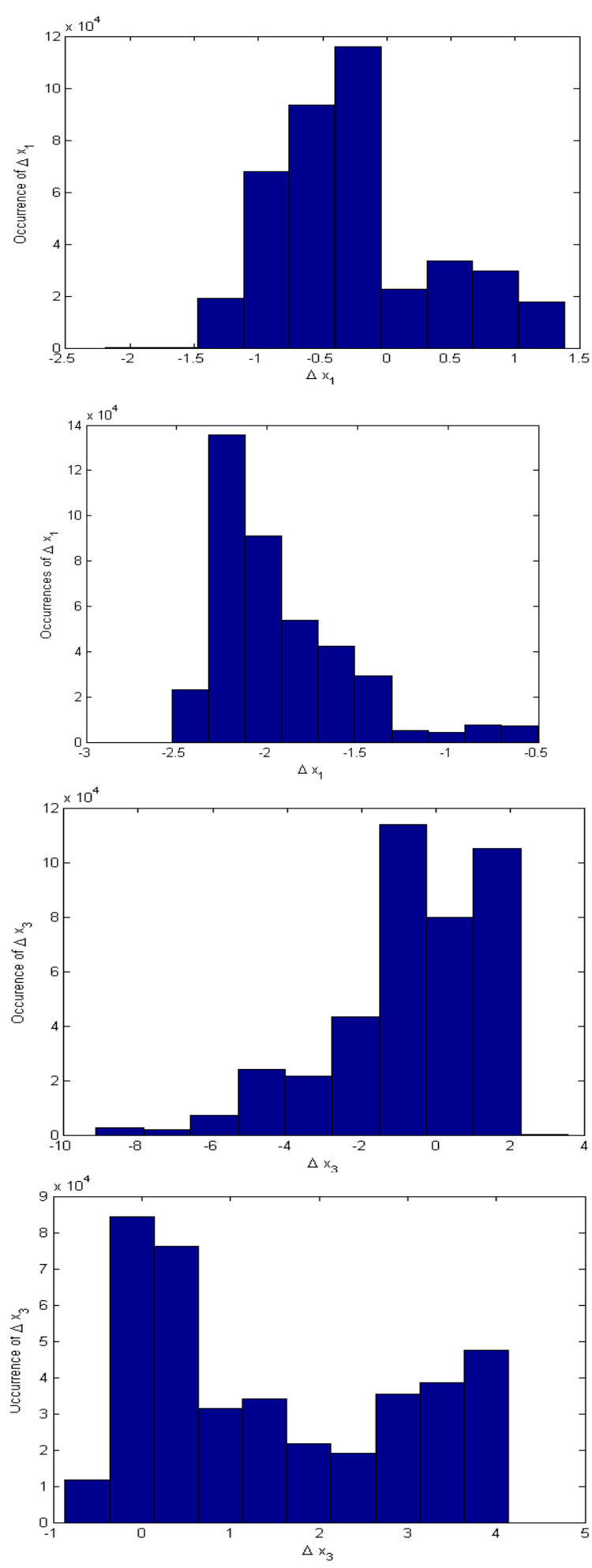

Fig. 3. The chaotic Hitzl-Zele map for initial conditions vector $\left.x_{1}(0), x_{2}(0), x_{3}(0)\right)=(0.65,0.17,0.7)$. The distribution of the determinant corresponding to the observable $x_{1}$ and $x_{3}$ for $(a, b)=(0.275, b=$ $0.87)\left(2^{n d}\right.$ and $\left.4^{t h}\right)$ and $(a, b)=(-0.69,0.85)\left(1^{\text {st }}\right.$ and $\left.3^{r d}\right)$.

Nevertheless, in a physical system, the noise superposed on the signals leads to a practical impossibility to reconstruct the states of the transmitter, once the absolute value of

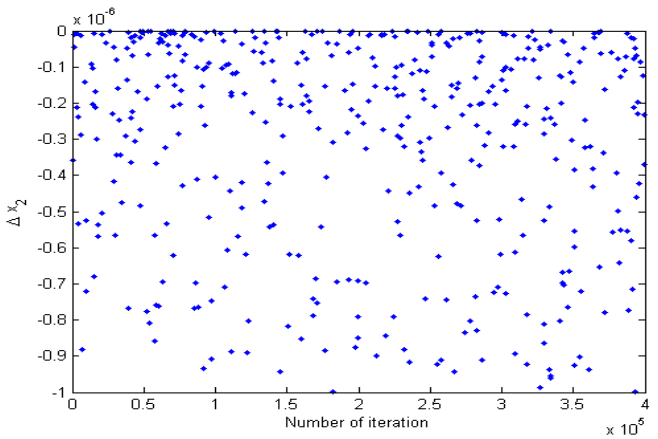

Fig. 4. The chaotic Hitzl-Zele map for initial conditions vector $\left.x_{1}(0), x_{2}(0), x_{3}(0)\right)=(0.65,0.17,0.7)$, parameters $(a, b)=$ $(0.275, b=0.87)$. The determinant $\Delta x_{2} \in\left(-10^{-6}, 10^{-6}\right)$.

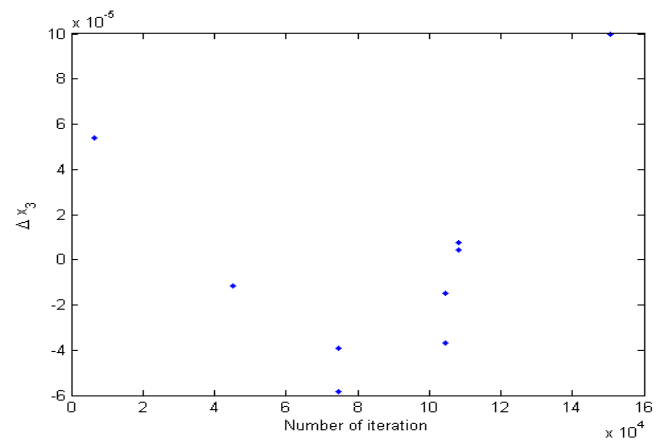

Fig. 5. The chaotic Hitzl-Zele map for initial conditions vector $\left.x_{1}(0), x_{2}(0), x_{3}(0)\right)=(0.65,0.17,0.7)$, parameters $(a, b)=$ $(0.275, b=0.87)$. The determinant $\Delta x_{3} \in\left(-10^{-4}, 10^{-4}\right)$.

the determinant of the observability matrix is smaller than the noise. Therefore, one cannot reconstruct the original system while the absolute value of the determinant of the observability matrix is smaller than the incertitude on the signals.

Observed by the state $x_{3}$, despite that the singularity can not be crossed by the dynamics of the system, Fig.6 shows that very often the value of $\Delta x_{3}$ is very close to zero. To better appreciate this proximity, we magnifyied the vertical scale (Fig.7). We find that for approximately $30 \%$ of the duration of the common example, the observability singularity of the system is approached better than $10^{-7}$. Thus, despite the fact that the singularity is not reached, the system seems much more suitable to a data encryption with loss of observability that the previously studied discrete recurrence.

\section{CONCLUSIONS}

After describing the concept of observability singularities, we detailed their existence in the case of a discrete nonlinear recurrence and a chaotic continuous system, very used in the field of chaotic encryption. We show that these singularities form a plan in the phase space of dimension 3. Except the case when being collinear, in the continuous time case, the intersection of a line and a plane provides a point and corresponds to a zero duration and in the discrete time case the probability that the dynamics falls exactly in the 


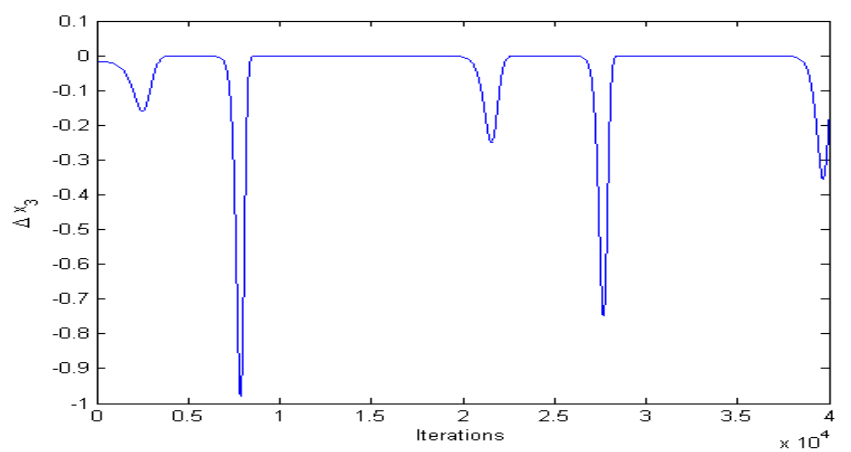

Fig. 6. The Colpitts oscillator. The evolution of the determinant of the observabiliy matrix, with the observable $x_{3}$ for the strange attractor engendered by parameters $g=4.46$ and $Q=1.38$, initial conditions $x_{1}(0)=0.9134, x_{2}(0)=0.6324, x_{3}(0)=0.0975$.

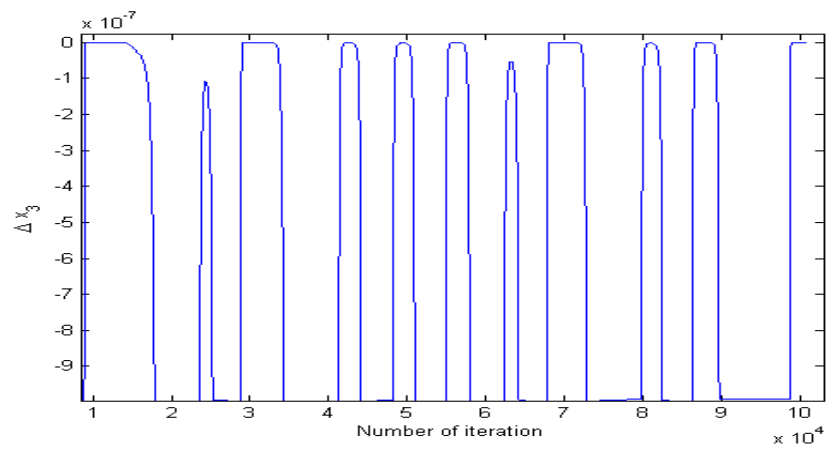

Fig. 7. The Colpitts oscillator. The evolution of the determinant of the observabiliy matrix, with the observable $x_{3}$ for the strange attractor engendered by parameters $g=4.46$ and $Q=1.38$, initial conditions $x_{1}(0)=0.9134, x_{2}(0)=0.6324, x_{3}(0)=0.0975$.

singularity sets is very weak. Nevertheless, the imprecision of numerical calculations or the noise on analog signals prohibit the reconstruction of the state vector as the determinant of the observability matrix is close to zero. Consequently, the duration of non-observability is substantially increased and can be, thus, used to secure the data transmission. The observability singularities depend on the parameters of the systems. It is, then, possible to take these parameters as encryption key, and frequently change them, which prevents the attacker to know when the system is observable or not. This involves an increase of the duration during which the system is not observable, in order to ensure synchronization loss. This is a challenge to come.

\section{REFERENCES}

[1] J-P Barbot, D. Boutat and T. Floquet, An observation algorithm for nonlinear systems with unknown inputs, in Automatica V 45, N8, pp. 1970- 1974, 2009.

[2] L. Boutat-Baddas, J.P. Barbot, D. Boutat and R. Tauleigne, Sliding mode observers and observability singularity in chaotic synchronization, Mathematical Problems in Engineering, 2004.

[3] S. Diop and M. Fliess, Nonlinear observability, identifiability and persistent trajectories, in Proc. 30th IEEE Conf. Decision and Control, pp. 714-719, 1991.

[4] M. Frunzete, A. Luca, A. Vlad, J.-P. Barbot, Observability and singularity in the context of the Rossler map, U.P.B. Sci. Bull., Series A, Vol. 74, Iss. 1, 2012 ISSN1223-7027.
[5] M. Frunzete, J.-P. Barbot, C. Letellier, Influence of the singular manifold of non observable states in reconstructing chaotic attractors, Physical Review E 86, 2, 2012.

[6] J.P. Gauthier, H. Hammouri, S. Othman, A simple observer for nonlinear systems applications to bioreactors, Automatic Control, IEEE Transactions on 37 (6), 875-880.

[7] G. Grassi and D. A. Miller, Theory and Experimental Realization of Observer-Based Discrete-Time Hyperchaos Synchronization, in IEEE Transactions on Circuits and SystemsI: Fundamental Theory and Applications, vol. 49, no 3. 2002.

[8] R. Hermann and A. Krenner, Nonlinear controllability and observability, in IEEE Transactions on Automatic Control VOL. AC-22, No. 5, 1977.

[9] M. L'Hernault, J.-P. Barbot, A. Ouslimani, Feasility of Analog Realization of a Sliding-Mode Observer: Application to Data Transmission, in Circuits and Systems I: Regular Papers, IEEE Transactions on (Volume:55 , Issue: 2 ), pp. 614 - 624, ISSN : 1549-8328, 2008.

[10] A. Isidori, Nonlinear Control Systems, Third Edition, Springer, ISBN: 3-540-19916-0 New York, 2001.

[11] V. Katz, A History of Mathematics (Brief ed.), Pearson Education, pp. 378379, 2004.

[12] C. Letellier, L. A. Aguirre and J. Maquet, Relation between observability and differential embeddings for nonlinear dynamics Physical Review E, 71, 066213, 2005.

[13] C. Letellier, R. Gilmore, and L. A. Aguirre, Robustesse dune reconstruction du portrait de phase et observabilité, Rencontre du nonlinéaire, 2007.

[14] G.M. Maggio, O. De Feo, M.P. Kennedy, Nonlinear analysis of the Colpitts oscillator and applications to design, IEEE Transactions on Circuits and Systems- I: Fundamental Theory and Applications, vol. 46, no. 9, 1999.

[15] W. Perruquetti and J.-P. Barbot, Chaos in automatic control, CRC Press, Taylor\& Francis Group, 2006.

[16] Respondek W, Right and Left Invertibility of Nonlinear Control Systems, in Nonlinear Controllability and Optimal Control, ed., Sussmann H. J. (Marcel Dekker, New York) 24: 133-176, 1990.

[17] P. Saha, S. H. Stragatz, The birth of period three, in Mathematics Magazine, vol. 68, no. 1, pp. 42-47, 1995.

[18] G. Zheng, M. Ghanes, and J.-P. Barbot, Secure data trasnmission based on multi-input multi-output delayed chaotic system. 